\title{
Modes de gestion des terres dans la zone tampon de la réserve de biosphère de la Pendjari
}

\author{
Romaric S. LOKOSSOU 1, Gaston S. AKOUEHOU 1, Mickaël L. AVONONMADEGBE 2, Augustin OROU \\ MATILO ${ }^{1 .}$ \\ ${ }^{1}$ Centre d'études de recherches et de formations forestières, ministère en charge de l'environnement, Bénin. \\ 2 Département d'Aménagement de Gestion de l'Environnement Faculté des sciences agronomiques, université \\ d'Abomey-Calavi, Bénin. \\ Auteur correspondance e-mail : lokossouromaric@yahoo.fr
}

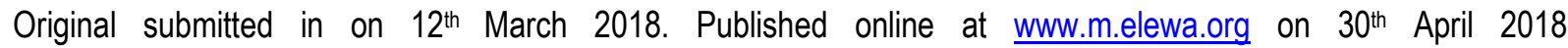
https://dx.doi.org/10.4314/jab.v124i1.5

\section{RESUME}

Objectif : La présente étude relative à l'impact des méthodes de gestion des terres riveraines mises en œuvre dans le parc de la Pendjari vise à mettre en évidence les conséquences des pratiques mises en œuvre sur la conservation dudit parc.

Méthodologie et résultats : Pour ce faire, une enquête a été réalisée et portait sur 130 producteurs dans neuf (9) villages riverains du parc, sur les deux axes Tanguiéta-batia et Tanguiéta porga stratifié autour du parc. Elle s'est focalisée sur les systèmes de culture observés et leur impact sur la conservation des écosystèmes. Le traitement des données a été effectué sur la base d'une matrice brute de données sur 130 producteurs riverains. Dans un premier temps, la matrice brute a été soumise à un AFC (Factors Components Analysis), une classification hiérarchique, des tests de variance tels que le test non-paramétrique de kruskal wallice et les tests d'indépendance de khi 2 (X2). Les principales menaces à la conservation des ressources du parc identifiées sont la dégradation physique des sols due à l'utilisation de machinerie lourde (tracteur) entraînant le compactage du sol et une diminution de leur porosité et également la dégradation chimique des sols due à l'utilisation croissante de surdoses d'engrais minéraux (chimiques) provoquant l'acidification et l'asphyxie du sol. II ressort également de l'analyse socio-économique que les catégories de producteurs les plus menaçantes pour la conservation de la biodiversité dans cette réserve sont, d'une part, les producteurs de l'axe Tanguiétaporga (1ère auréole) qui ont largement adopté la culture du coton et la mécanisation avec l'utilisation de tracteurs qui ont un impact négatif sur les sols. En outre, ils menacent la biodiversité en convertissant l'écosystème forestier en terres agricoles.

Conclusion et application des résultats : Les principales menaces qui pèsent sur les écosystèmes autour du parc de la Pendjari sont la dégradation physique et chimique qui est principalement due à l'adoption généralisée de la culture du coton qui induit l'utilisation de machines lourdes et de produits chimiques. Ainsi, la prise en compte de ces menaces par l'organe de gestion du parc (CENAGREF-Centre National de Gestion des Réserves de Faune) et l'adoption d'une agriculture plus biologique pourrait améliorer la conservation de cet écosystème forestier. Dans un but d'approfondissement, une étude sur la qualité des eaux (rivière Pendjari et mares) du parc devrait être envisagée pour évaluer l'état de l'écosystème suite aux activités anthropiques, gage de la conservation de la faune sauvage.

Motsclés: écosystème forestier, systèmes de culture, parc Pendjari, agriculture extensive, 


\section{ABSTRACT}

Objective: The present study related to the impact of the riparian land management methods implemented in the Pendjari Park aims to highlight the various consequences of these implemented methods or technics on this Park ecosystem conservation.

Methodology and Results : To achieve this, a survey was carried out in nine (9) villages bordering the park on the two Tanguiéta-batia and Tanguiéta porga axes laminated around the park and concerned 130 producers. They focused on the observed cropping systems and their impact on ecosystem conservation. Data processing was done on the basis of a raw matrix of data from 9 surveys. As a first step, the raw matrix was subjected to an AFC (Analysis Factors Components), a Hierarchical Classification, a tests of variance such as the nonparametric test of kruskal wallice and the tests of independence of khi $2(x 2)$ in order to highlight the typology of the producers and the different relations among them. It shows that riparian populations greatly influence the sustainable conservation of the reserve through practices such as: the high rate of mineral fertilization, short fallow periods, extensive agriculture and illegal exploitation of Park resources. The main threats to the conservation of park resources identified are the physical degradation of soils due to the use of heavy machinery (tractor) causing soil compaction and a decrease in their porosity. Also chemical soil degradation due to the increasing overdose use of mineral (chemical) fertilizers causing acidification and soil asphyxiation. It is also apparent from the socio-economic analysis that the most threatening categories of producers for the conservation of biodiversity in this reserve are, on the one hand, the producers of the Tanguiéta-porga (1st halo) axis who have largely adopted Cotton cultivation and mechanization that induces the use of tractors that have a negative impact on soils. In addition, they threaten biodiversity by converting large forest ecosystem to agricultural land.

Conclusion and application of the results : The main threats to ecosystems around the Pendjari Park are the physical and chemical degradation that is mainly due to the widespread adoption of cotton cultivation which induces the use of heavy machinery and chemicals. Thus, the consideration of these threats by the park management office (CENAGREF) and the adoption of more organic agriculture could improve the conservation of this forest ecosystem. For deeper understanding of impacts, a water quality study of Pendjari river and related ponds of the park should be considered to assess the state of the ecosystem as a result of anthropogenic activities, a guarantee of wildlife conservation.

Keywords: Forest ecosystem, cropping systems, Pendjari Park, extensive agriculture,

\section{INTRODUCTION}

Les réserves de Biosphère dont la Pendjari sont des aires protégées terrestre, reconnues au niveau international dans le cadre du programme de I'UNESCO sur l'homme et la Biosphère (MAB) comme sites privilégiées pour la promotion et la démonstration des relations équilibrées entre les êtres humains et la nature (UNESCO 1995). Créée d'abord comme aire protégée à partir des années 50 puis réserve de biosphère depuis 1986, la réserve de la Pendjari n'a pas échappé à la pression humaine due au manque de moyens de contrôle et de suivi du Centre National de Gestion des Réserves de Faune (CENAGREF), surtout par l'absence d'une stratégie appropriée de gestion impliquant les populations riveraines (Tiomoko, 2007.). En effet, Deldicque (2007) indique que le paysan est le gestionnaire direct de son environnement de travail qui possède des connaissances de son milieu naturel. Dans ces conditions, son implication dans la gestion et la prise en compte de ses besoins, les retombées économiques et financières à son profit et la place de choix qu'il occupe dans le système de gouvernance sont autant d'éléments nécessaires pour faire face aux défis de la protection efficiente de la réserve. Selon Triplet et al (2009), la cogestion décrit une forme de partenariat par lequel toutes les parties prenantes intéressées s'accordent pour partager les fonctions de gestion, les droits et responsabilités sur une portion de territoire ou une gamme de ressources.Baldus (2001) a montré que la conservation de la diversité biologique coûte cher aux pays africains qui avec leurs ressources 
limitées, doivent faire face aux défis du développement. Or il est urgent selon la FAO, de favoriser des interactions positives entre ces deux utilisations des terres (agriculture et forêt) vu que ces écosystèmes forestiers sont aussi importantes que l'agriculture pour l'homme et pour l'avenir de la planète (FAO, 2016). D'où l'intérêt de ce travail se justifie par le fait de l'augmentation de la pression démographique humaine et l'occupation des terres

\section{MATÉRIEL ET MÉTHODES}

Milieu d'étude : La Réserve de Biosphère de la Pendjari (RBP) est située au Nord-Ouest de la République du Bénin, plus précisément dans le Département de l'Atacora, sur les territoires des communes de Tanguiéta, Matéri et de Kérou. Elle couvre une superficie de 477.802 ha et ces limites géographiques sont comprises entre $10^{\circ} 30^{\prime}$ et $11^{\circ} 30^{\prime}$ de latitude Nord, $0^{\circ} 50^{\prime}$ et $2^{\circ} 00^{\prime}$ de longitude Est plus précisément dans le Département de l'Atacora, sur les territoires des communes de Tanguiéta, Matéri et de Kérou (CENAGREF, 2009 ; et Djafarou, 2014.). La RBP, un des plus grands écosystèmes du pays a été classé le 13 décembre 1954 en forêt domaniale et en réserve partielle de faune de la boucle de la Pendjari. Son statut actuel de Parc National de la Pendjari a été fixé par le décret N¹32/FR/MAC/CF du 6 mai 1961 qui le classe comme une zone protégée avec interdiction des droit d'accès et d'usage aux de la zone tampon (Sogbohossou, 2008). II s'agit spécifiquement de i) Décrire les différentes relations de production avec l'aire protégée a travers l'analyse des systèmes de production ; ii) Caractériser sur le plan socio-culturelles les catégories de producteurs sur la conservation du Parc ; iii) évaluer l'influence de la gestion des terres agricoles riverains sur le maintien de l'écosystème de la réserve de Biosphère Pendjari

populations locales. Elle comprend : le Noyau central (266.040 ha) ; la Zone Cynégétique de la Pendjari (170.080 ha) ; la Zone Cynégétique de l'Atacora (125.640 ha) ; la Zone d'occupation contrôlée (ZOC) ; la Zone de Transition essentiellement composée des terroirs villageois. La Réserve de biosphère de la Pendjari (RBP) fait partie du plus grand ensemble d'aires protégées d'Afrique de l'Ouest, le complexe WAP (W-Arly-Pendjari), qui comprend aussi la Réserve de biosphère transfrontalière W (Bénin-Niger-Burkina Faso), et plusieurs aires protégées de statuts divers au Burkina Faso (Réserve partielle de Pama, Réserve totale de faune d'Arly, Réserve du Singou) et au Togo (Parc national de la Kéran, réserve de faune Oti-Mandouri), sur une superficie totale d'environ $50000 \mathrm{~km} 2$. La RBP couvre environ $5000 \mathrm{~km} 2$, soit $10 \%$ de l'ensemble.

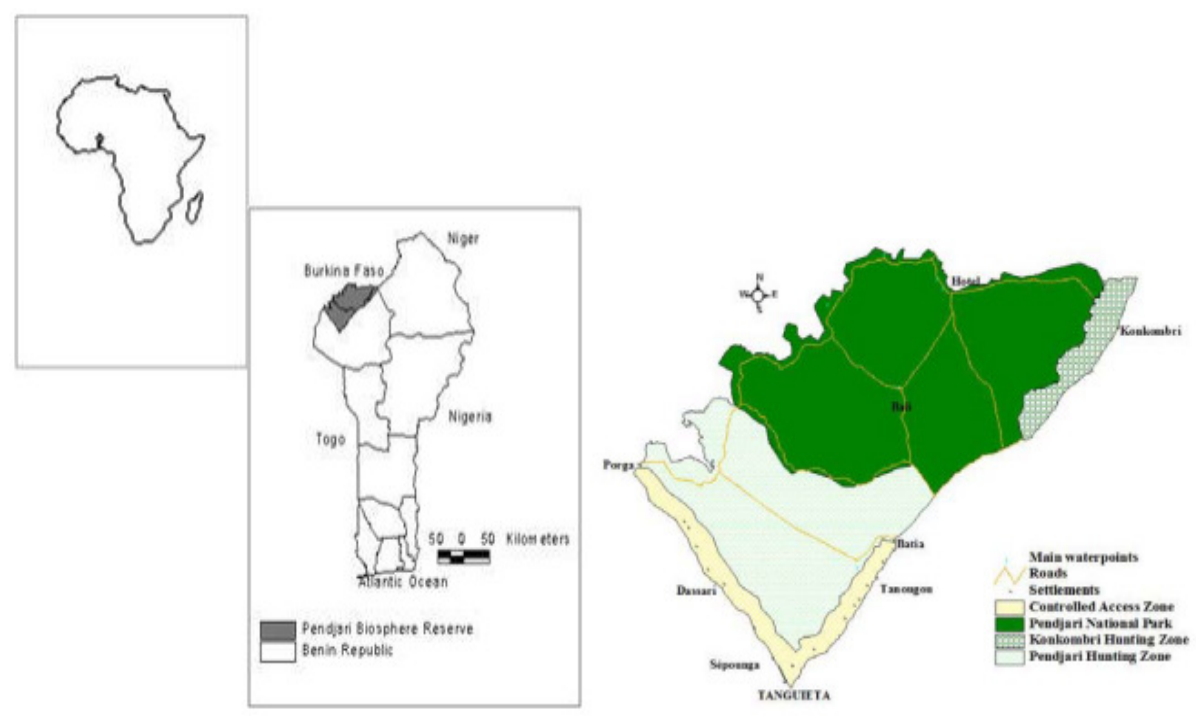

Figure1 : Carte de zonage de la Réserve de Biosphère de la Pendjari 
La réserve de la biosphère Pendjari est sous le climat est de type soudano- guinéen sec influencé par la chaîne de l'Atacora. II est connu sous le nom de " climat atacorien » caractérisé par des températures plus faibles que dans l'ensemble de la zone soudanienne (PAVICO, 2002; AOC, 2006). Les orages sont fréquents et ce climat est caractérisé par une pluviométrie uni- modale, une saison pluvieuse d'Avril à Octobre et une saison sèche allant de novembre à mars. Les températures moyennes mensuelles ne descendent pratiquement pas en dessous de $20^{\circ}$, les écarts mensuels restent faibles $\left(36^{\circ}\right.$ en mars au plus chaud et $24^{\circ}$ en Août au plus frais). La pluviométrie annuelle moyenne est $1100 \mathrm{~mm}$ et les précipitations sont irrégulièrement réparties tout au long

\section{MATERIEL ET METHODE D'ETUDE}

Collecte des données : La collecte d'informations a été réalisée à travers des entretiens de groupe, des focus groupe, entretiens individuels (questionnaires) et des observations personnelles au niveau des populations riveraines. Les informations collectées sont relatives aux problèmes de gestion des terres agricoles et le rôle de l'administration du Parc de Pendjari; la caractérisation des systèmes culturaux et catégories de producteurs observés et enfin les influences directes et indirectes des pratiques agricoles sur la conservation de la biodiversité. Les enquêtes ont été effectuées suivant les deux axes cidessous et les villages ont été choisis sur chaque axe suivant une stratification qui a tenu compte de la distance

Tableau 1: répartition des producteurs suivant les auréoles de la saison pluvieuse, ce qui constitue un frein pour le développement de l'agriculture. La région est soumise à l'harmattan, vent sec et chaud qui souffle entre les mois de novembre et février. A partir de fin mars Avril, la région est sous l'influence de la mousson océanique qui apporte la pluie d'Avril à Octobre comme le montre la figure de son diagramme climatique. La période sèche est définie comme étant les mois où la pluviométrie est inférieure à la moitié de l'évapotranspiration potentielle. On note ainsi, dans le milieu d'étude, deux saisons à savoir une saison pluvieuse et une saison sèche. La saison sèche dure cinq mois de mi- avril à mi- octobre et la saison pluvieuse occupe le reste de l'année.

séparant ces villages de la limite du Parc. Ainsi l'étude a retenu neuf (9) villages sur les deux axes :

- Sur l'axe tanguiéta-batia: Tchanwassaga, Bourgnissou, Nanébou, Tanongou Batia

- Sur l'axe tanguiéta-porga: Tiélé, Dassari, wantéou et Porga

L'échantillonnage a été aléatoire et cent trente producteurs (130) repartis suivant les villages cités plus haut (tableau 1) ont été enquêté sur un total de 40.000 riverains qui influencent directement les ressources du Parc (enquête PNUD 2000). La fréquence d'échantillonnage est de 0.00325 soit $325 / 100000$.

\begin{tabular}{|l|l|l|l|}
\hline Auréoles & Nombre de kilomètres & Villages & $\begin{array}{l}\text { Nombre } \\
\text { de producteurs }\end{array}$ \\
\hline Auréole 1 & {$[1,2[$} & Batia, porga dassari et tanougou. & 82 \\
\hline Auréole 2 & {$[3,5[$} & $\begin{array}{l}\text { Wantéou, bourgnissou, } \\
\text { tchanwassaga, tanougou, tiélé. }\end{array}$ & 39 \\
\hline Auréole 3 & {$[6,7[$} & Tiélé nanébou. & 09 \\
\hline
\end{tabular}

Source : données enquêtes Avononmadegbe 2015

Caractérisation et impact socio-culturelles des catégories de producteurs sur la conservation du Parc: Les données collectées sont relatives aux différentes pratiques agricoles dans la zone tampon et aux influences directes et indirectes de ces pratiques sur l'écosystème du Parc. Une caractérisation des systèmes culturaux, des différents groupes socio-culturelles des producteurs ont été faites suivant les auréoles autours de la réserve afin d'analyser leur impact sur la conservation de l'écosystème du Parc Pendjari. Le traitement des informations issues des enquêtes a permis une illustration théorique des données collectées. Les analyses ont été faites sur la base d'une matrice brute de données de 9 relevés. Chaque relevé est caractérisé par un village et son score face aux 31 variables liées aux producteurs, leurs systèmes culturaux et leurs impacts sur la conservation. Dans un premier temps, la matrice de neuf relevés constitué par chaque village d'enquête a été soumise à une AFC (Analysis Factor Components) afin de décrire les relations existantes entres les 9différents 
villages d'enquêtes et les 32 variables en vue de la caractérisation des producteurs. Ensuite, la même matrice brute a été à nouveau soumise à une Classification Hiérarchique en vue de catégoriser les différents groupes socioéconomiques et d'impacts. Des tests statistiques notamment celui d'indépendance de Khi-2 et celui de comparaison de proportions ont été effectués pour appuyer les analyses factorielles. Au cours

\section{RÉSULTATS}

Relation socio-culturelles des catégories de producteurs : II ressort de l'analyse socioculturelle des relations de production que :En général, les producteurs ont adoptés les cultures de rente dont le coton et certaines cultures vivrières comme le mil, le maïs, le sorgho, l'igname, l'arachide et le voandzou. Ils emblavent de grandes superficies et pratiquent très souvent une agriculture mécanisée. Ils ont une préférence pour les engrais minéraux qu'ils utilisent en grande quantité et obtiennent de bons rendements. Ces producteurs se rencontrent le plus souvent sur l'axe tanguiéta-porga et plus particulièrement dans le village de Porga. Sur ce même axe, dans les villages de Tiélé et Dassari qui de l'analyse, les informations issues des 130 enquêtés ont été réparties comme suit: des grands producteurs (dont la superficie emblavée est supérieure à dix (10) hectares); des producteurs moyens (dont la superficie emblavée est entre six (6) et dix (10) hectares) et des petits producteurs (dont la superficie emblavée est inférieure ou égale à cinq (5) hectares).

cultivent le mil ont tendance à ne plus cultiver d'autres cultures vivrières (l'arachide, le maïs ni le riz). Par ailleurs, ceux qui cultivent l'igname, pratiquent de longue durée de jachère et sont pour la plupart des producteurs de la deuxième auréole d'étude du village de tchawansaga nanébou tanougou et bourgnissou, chez d'autres producteurs de la même auréole dans les ménages de grande taille les femmes produisent du riz (wantéou). Ces producteurs, de la première auréole, surtout ceux de tanougou ont une préférence pour les engrais organiques et exploitent les plus les ressources de la réserve (Tanougou et Bourgnissou.

\section{CA factor map}

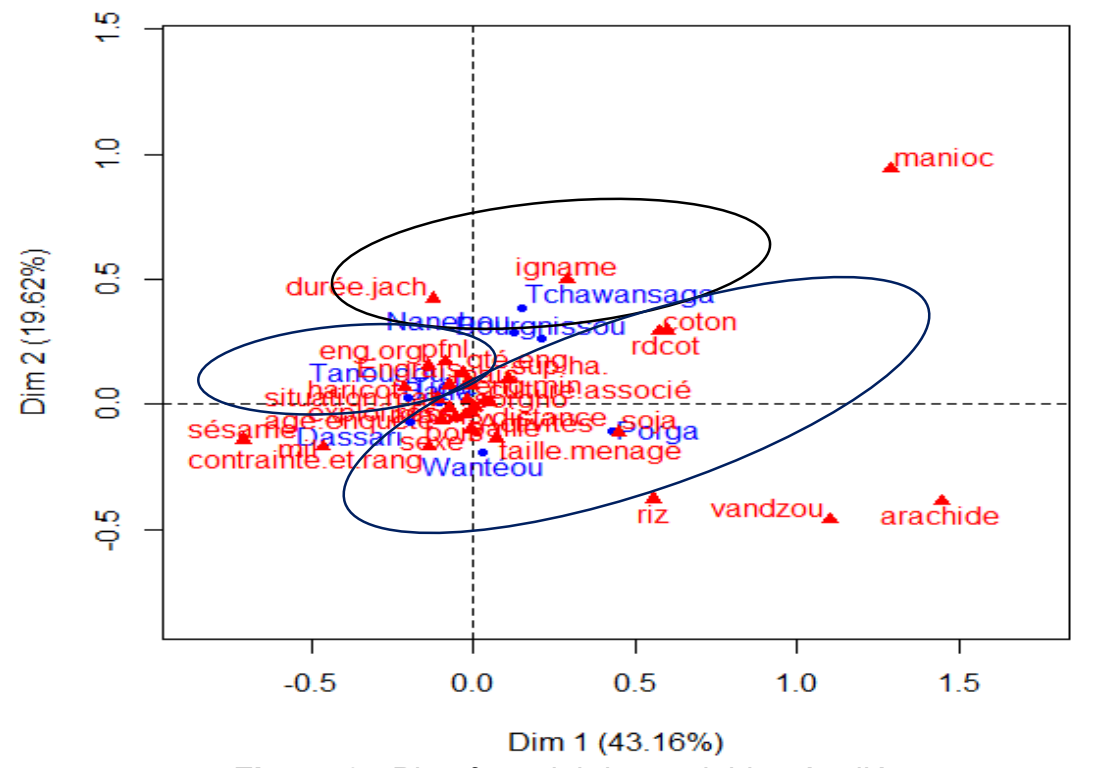

Figure 3 : Plan factoriel des variables étudiées

Légende : age.enquèté : âge des enquêtés ; Sexe : sexe des enquêtés; situation.mat: situation matrimoniale de l'enquêté; taille. ménage: taille du ménage de l'enquêté; Activités: activités pratiqués par l'enquêté; sup. ha: superficie en hectares emblavée par l'enquêté; durée.jach : durée de la jachère pratiquée par l'enquêté; culture. associé : pratique d'association de culture; Engrais : Utilisation d'engrais; qté.eng : quantité d'engrais utilisée; eng.min : utilisation d'engrais minéraux;eng.org : utilisation d'engrais organique; rdcot : rendements du coton; rdmais : rendements du maïs; contrainte.et.rang : contrainte observée par enquêté; Distance : distance des champs des enquêtés par rapport à la limite de la réserve; exploi.ress: exploitation des ressources dans la réserve; Bois : exploitation de bois dans la réserve; Pfnl : exploitation de produits forestiers non ligneux dans la réserve; Maïs: culture du maïs par les enquêtés ;Mil: culture du mil par les enquêtés; Coton : culture du coton par les enquêtés; Arachide : culture de 
l'arachide par les enquêtés; Igname : culture de l'igname par les enquêtés; Sorgho : culture du sorgho par les enquêtés; Haricot : culture de l'haricot par les enquêtés; Riz : culture du riz par les enquêtés ; Sésame : culture du sésame par les enquêtés; Soja : culture du soja par les enquêtés; Voandzou : culture du voandzou par les enquêtés; Manioc : culture du manioc par les enquêtés

La classification Hiérarchique a révélé (Figure 4), à un seuil de similarité de $50 \%$ trois grands (03) catégories de producteurs suivant l'analyse factorielle des composantes des relevés des neuf(9) villages d'étude (Figure 4). La première catégorie regroupe les producteurs qui, pratiquent de longue durée de jachère et qui cultivent en majorité l'igname. II s'agit des exploitants de l'axe tanguiéta -batia (1ère auréole) plus précisément des villages de tchawansaga nanébou tanougou et bourgnissou qui sont plus proches de la réserve et plus éloignés de la route inter état. Ce sont des producteurs moyens ayant une préférence pour les engrais organiques. La deuxième catégorie est composée d'une part importante des producteurs ayant adoptés une agriculture itinérante sur brulis préférant la culture du mil aux autres cultures. La plupart de ces producteurs sont des femmes, emblavant peu de superficie et sont répartis sur les deux axes dans les $2^{\mathrm{ème}}$ et 3 ème auréoles mais dominant dans le village de tiélé ( $3^{\text {ème }}$ auréole). Au niveau de la troisième catégorie, ce sont les producteurs qui utilisent de fortes doses d'engrais minéraux et obtenant bons rendements pour le coton. II s'agit des exploitants de l'axe tanguiéta -porga (1ère et $2^{\mathrm{ème}}$ auréole). Ce sont des producteurs ayant adoptés un fort niveau de mécanisation et cultivant le coton. Le dégré de menace qu'ils constituent est surtout lié à leurs grand nombre dans la forêt car pour la plupart de petits producteurs, ils sont en quêté de terre et emblavent déjà des superficicies dans la limite de la réserve. Ils sont répartis sur les deux axes dans les deux premières auréoles mais dominent sur l'axe tanguiéta-porga et précisément dans les villages de porga et dassari (1 êre auréole) riverains à la réserve.

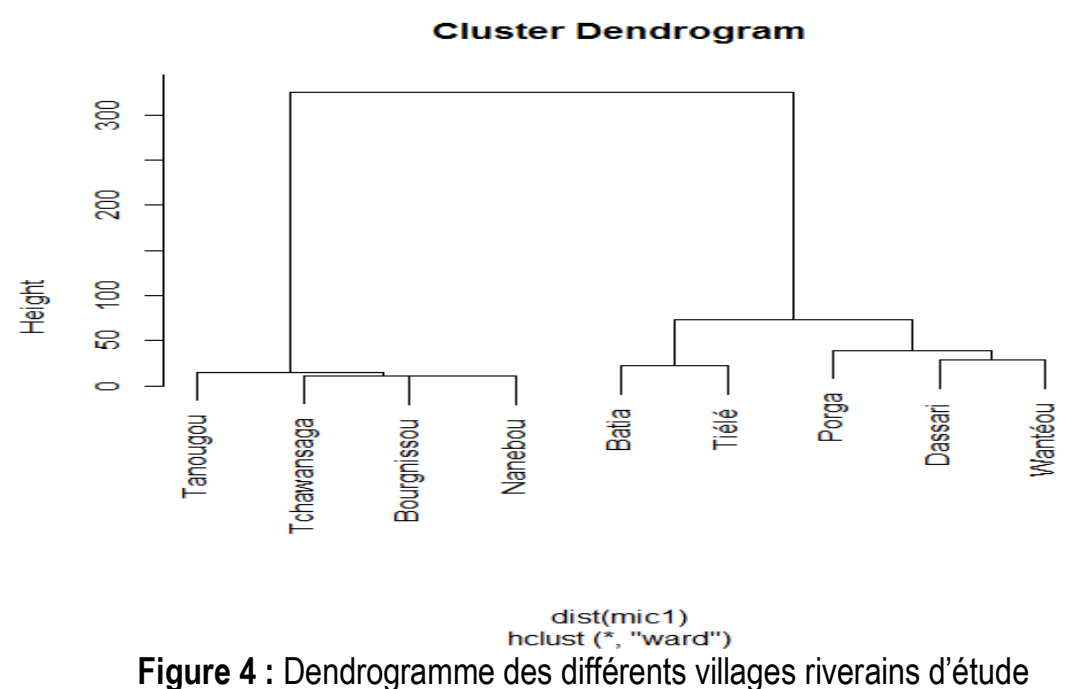

Influence des activités sur la biodiversité suivant les auréoles : La figure 5 montres la répartition des producteurs en fonction des distances qui les séparent des champs c'est-à-dire les auréoles d'études. 


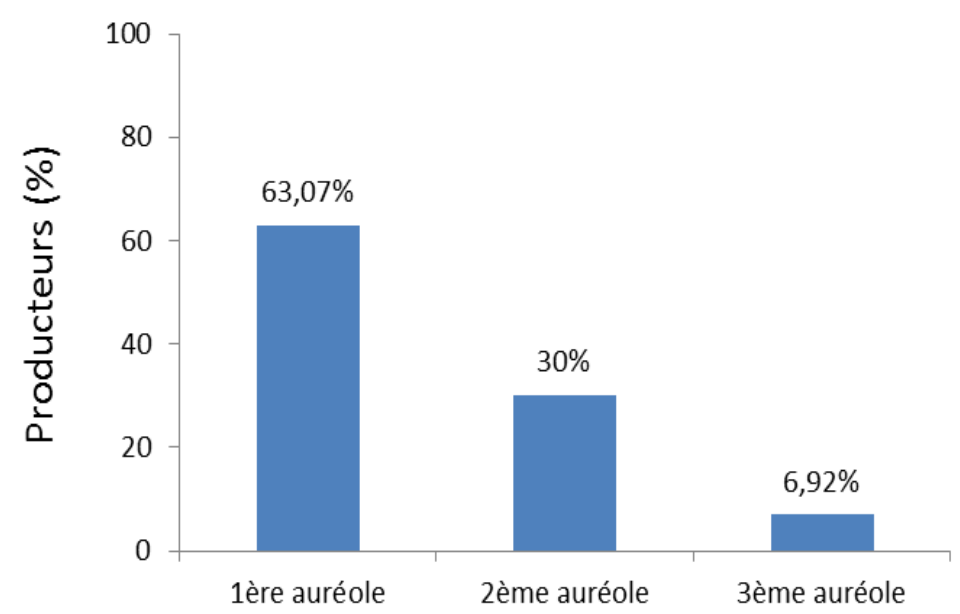

Figure 5: producteurs répartis en fonction de la distance entre les champs et la limite de la réserve.

Les données illustrées par le graphe de la figure 5 présenteraient une répartition des producteurs en fonction de la distance entre leurs champs et la limite de la réserve. La première auréole ( $63 \%$ des enquêtés) se situe entre un rayon de 1 à $2 \mathrm{~km}$ autour de la limite de la réserve. Elle contiendrait les villages de Batia, Porga, Dassari et Tanougou. La deuxième auréole $(30 \%$ des enquêtés) pourrait se situer dans un rayon de 2 à $5 \mathrm{~km}$ autour de la limite de la réserve. Elle a pris en compte les villages de Wantéou Bourgnissou et Tchanwassaga. La dernière auréole ( $7 \%$ des enquêtés) se situe dans un rayon de 5 à $7 \mathrm{~km}$ autour de la limite de la réserve et prend en compte les villages de Tiélé et Nanébou. Nous avons ainsi trois couches à niveau décroissant de menaces. Cette stratification a permis d'apprécier l'exploitation des ressources par zone et d'identifier l'intensité des impacts. A cet effet, un test d'indépendance de KHI-2 effectué a montré le lien entre la proximité du parc et l'exploitation des ressources. Les résultats de ce test $\left(X^{2}=3.6821, d f=2, p\right.$-value $\left.>0.1586\right)$, ont montré qu'il n'y a pas de relation entre l'appartenance aux auréoles et le niveau d'influence sur la réserve au seuil de $5 \%$. Cela voudrait dire que le niveau de pression exercé sur les ressources naturelles du parc n'est pas fonction de l'auréole d'appartenance. Aussi bien les populations des villages riverains qui sont proches que ceux qui sont loin de la forêt pourraient induisent les mêmes influences sur les ressources de la réserve forestière. Les informations du graphe pourraient alors être expliquées par la présence dominante des champs des producteurs dans les auréoles définies autour du parc. Les producteurs de la 1 ère auréole $(63 \%)$ ont leurs champs situés entre 1 et $2 \mathrm{~km}$ autour de la réserve. Ces producteurs exercent leurs pratiques agricoles à proximité de la réserve. Ceux des 2èmes et 3èmes auréoles ont leurs champs situés respectivement entre 3 et $5 \mathrm{~km}$ et entre 6 et $7 \mathrm{~km}$. La majorité des producteurs (93\%) enquêtés ont donc leur emblavure à moins de $5 \mathrm{~km}$ de la limite de la réserve. Ces derniers exploiteraient les ressources dans les limites de la réserve (fagots de bois, PFNL) dans la réserve pour répondre aux besoins d'alimentations, de logement a cause de la diminution des superficies emblavées suite a la pose des balises limitant la réserve.

Fertilisation des terres autour de la réserve: Le résultat du test relatifs à l'utilisation des types d'engrais par auréole khi-2 $\left(X^{2}=2.8589, d f=2, p\right.$-value $\left.>0.2394\right)$ a confirmé que l'utilisation du type d'engrais (minéraux ou organiques) n'est pas fonction de la distance des exploitations agricoles du parc ou de l'appartenance des utilisateurs (populations) aux auréoles. Indépendamment de l'appartenance des exploitations aux auréoles, les producteurs ont une tendance générale à utiliser en forte proportion des engrais minéraux plutôt que les engrais organiques. La figure 6 ci-dessous présente la répartition des producteurs en fonction du type d'engrais utilisé dans toutes les auréoles 


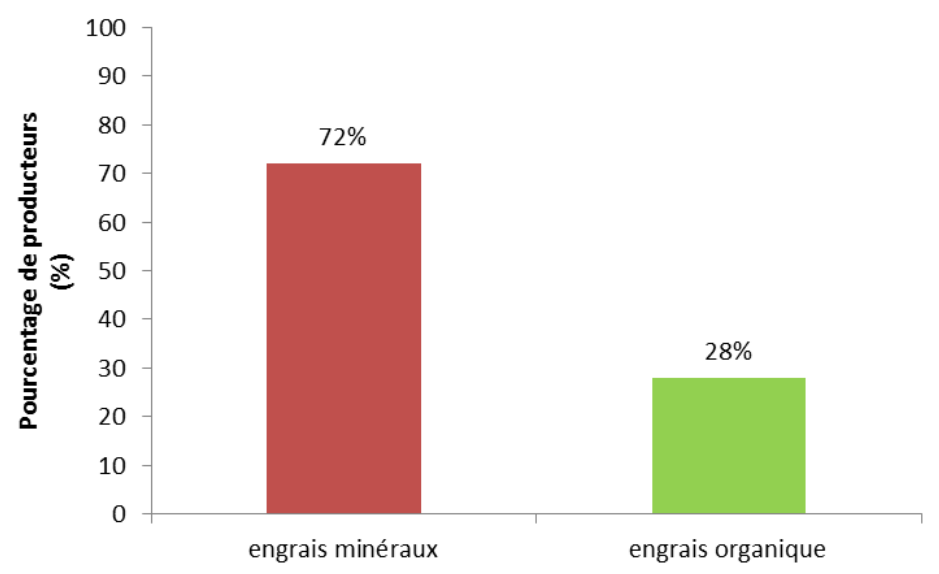

Figure 6: Répartition des producteurs en fonction du type d'engrais utilisé pour fertiliser leurs champs

L'analyse de la figure 6 a confirmé que les producteurs (proportion de $74 \%$ ) utiliseraient souvent les engrais minéraux pour fertiliser les sols. Ainsi, cette pratique dans les deux premières auréoles autour du Parc, épuiserait, les sols à long terme. Ce qui pourrait conduire à une dégradation chimique des sols autour de la réserve (acidification des sols).
Niveau de Mécanisation: Pour apprécier la relation qui existe entre la mécanisation et les différentes cultures emblavées autour de la Réserve de Pendjari, le test de kruskal wallice a été effectuée. Le résultat $d u$ test (Kruskal-Wallis chi-squared $=8.0958, \mathrm{df}=1, \mathrm{p}$-value $=0.004437$ ) nous révèle qu'il existe une relation permettant d'affirmer que la mécanisation est fonction de la culture emblavée.

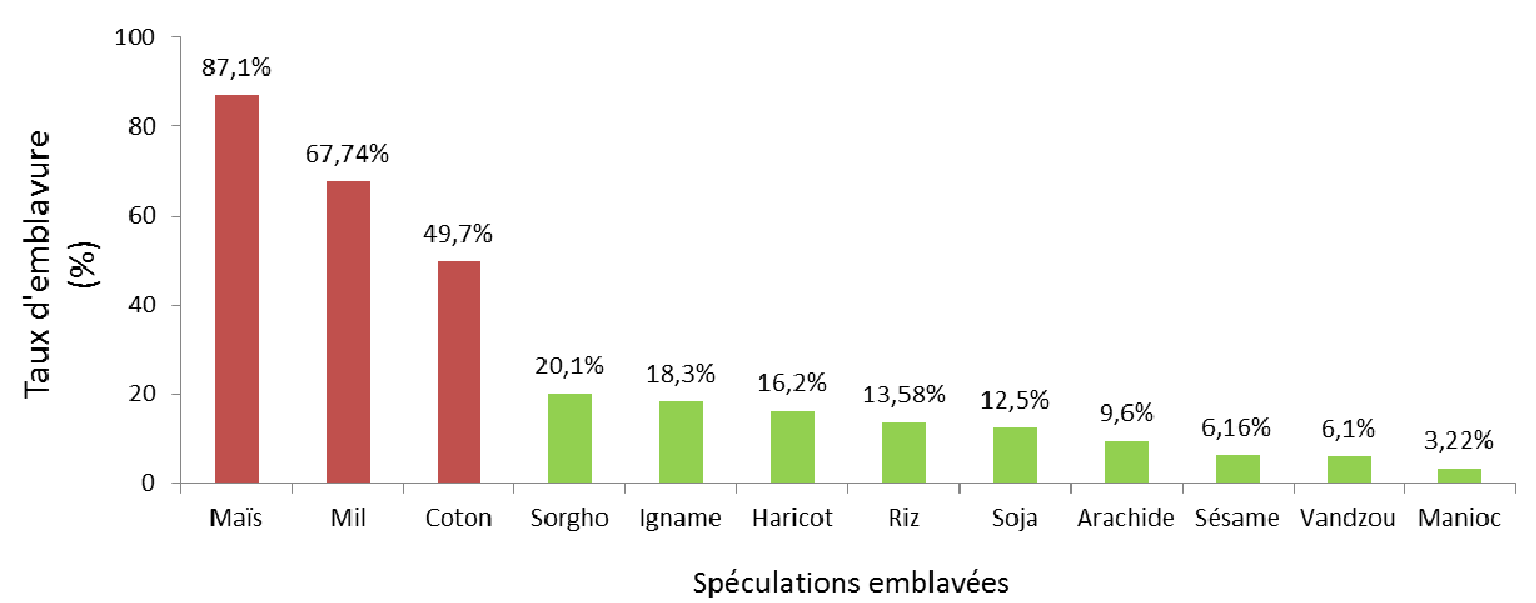

Figure 7: Taux d'emblavure des différentes spéculations cultivées

La figure 7 présente la proportion de producteurs cultivant chaque spéculation. On peut remarquer, que les producteurs cultivent majoritairement le maïs, le mil et le coton respectivement à des proportions de $87.1 \%$, $67.74 \%$ et $49,7 \%$. Ces cultures (coton et maïs), qui sont dominants, nécessitent le plus d'usage du tracteur entrainant un fort taux de mécanisation autour du Parc. $\mathrm{Ce}$ fort taux de mécanisation engendre des conséquences néfastes sur l'écosystème de la réserve et spécifiquement les sur sols. Ces cultures nécessiteraient de grandes superficies qui ne s'obtiennent qu'après destruction des terres forestières du Parc. Ainsi, le travail du sol au tracteur dominant dans la première auréole autour de la réserve, pourrait entrainer des dégradations physiques de l'état structural du sol par tassement, surtout lorsque le sol est humide au moment des opérations ; diminuant ainsi la porosité des sols et donc l'absorption de l'eau par les espèces végétales. 
Pratiques de Jachère : Les résultats du test khi- $2\left(X^{2}\right.$ $=11.3071, d f=4, p$-value $>0.02332$ ) viennent confirmer que la superficie emblavée est fonction de la proximité des producteurs de la réserve de la Pendjari. De l'analyse du résultat de ce test, il ressort que l'emblavure des superficies d'exploitation est en relation avec l'appartenance aux auréoles prédéfinies. Le constat serait que les deux premières auréoles qui sont les plus proches du parc, sont les zones d'intenses activités agricoles. Les agriculteurs de toutes les catégories y sont présents menaçant ainsi la biodiversité de la réserve forestière.

Tableau 2: répartition des types de producteurs par auréole

\begin{tabular}{|l|l|l|l|}
\hline Distance & grande superficie & superficie moyenne & petite superficie \\
\hline 1ère auréole & $2,43 \%$ & $13,41 \%$ & $84,14 \%$ \\
\hline 2ème auréole & $15,38 \%$ & $25,64 \%$ & 58,97 \\
\hline 3ème auréole & $11,11 \%$ & $22,22 \%$ & $66,67 \%$ \\
\hline
\end{tabular}
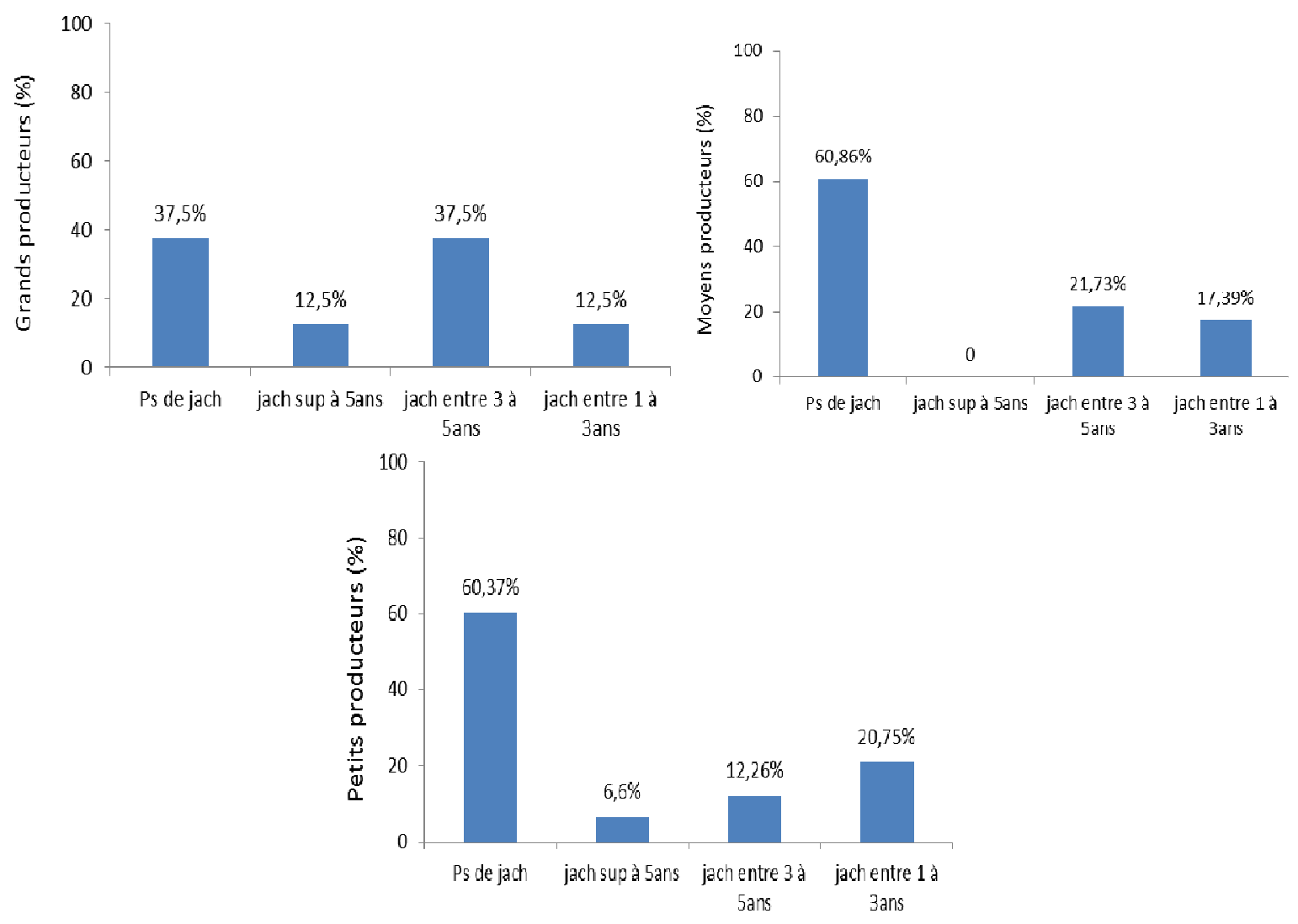

Figure 8 : Évolution de la superficie emblavée suivant les catégories de producteur

Légende : Ps de jach : pas de jachère ; jach sup à5ans : jachère supérieur à 5 ans ; jach entre 3 à 5 ans : jachère comprise entre

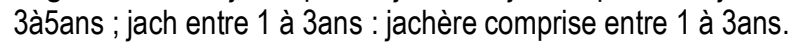

La Figure 8, qui présente la répartition des types de producteurs en fonction de la durée de jachère pratiquée pour les 3 catégories de producteur, vient confirmer les résultats des précédents tests. En moyenne plus de $60 \%$ des producteurs emblavant de petites et moyennes superficies et environ $40 \%$ de ceux emblavant de grande superficie(>10ha) ne pratiquent pas de jachère dans toutes les auréoles. Cela est dû selon eux au manque récurrent de terre dans les périphéries de la réserve. Ceux pratiquant les jachères, qui sont minoritaires chez les petits et moyens producteurs, les justifient par la pauvreté des sols qui chaque année demandent des 
doses supplémentaires d'engrais. D'une manière générale, à part ceux emblavant de grandes superficies (> 5ha) dont environ les $40 \%$ pratiquent une durée de jachère d'environ 3 à 5 ans, moins de $15 \%$ des producteurs réalisent de réelles jachères d'une période supérieure à 5 ans. Cela dans les premières auréoles autour du Parc ; ce qui influence la reconstitution des sols et par conséquent la conservation des terres de la réserve.

Traitements phytosanitaires : L'analyse de la présente figure a donné la répartition des producteurs selon l'usage de traitements phytosanitaires et a révélé qu'une proportion élevée $(78 \%)$ des producteurs fait usage de traitements phytosanitaires. Aussi les traitements phytosanitaires effectués à l'aide d'intrant chimique constitueraient-ils de véritable danger à la conservation des sols et à la santé des hommes et animaux de la réserve. Ils sont faits à l'aide de produits chimiques, polluant la nappe phréatique et par conséquent les eaux souterraines.

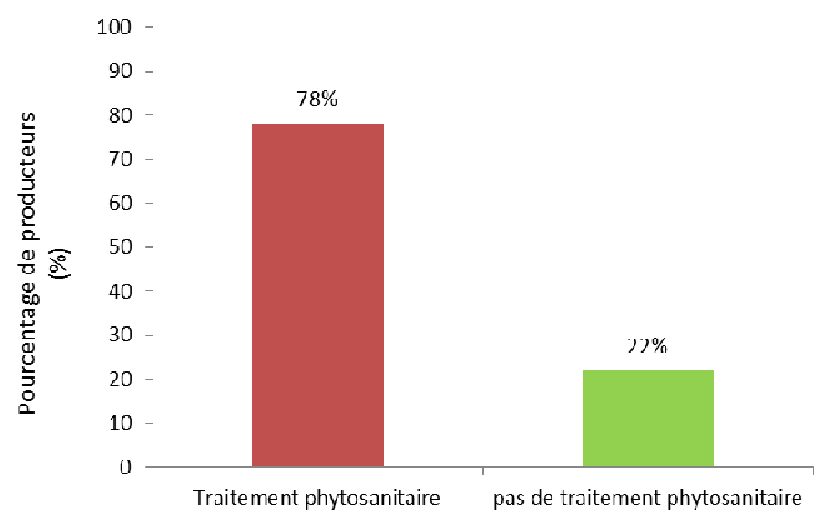

Figure 9: répartition des producteurs selon l'usage de traitements phytosanitaires

Le résultat de l'analyse des entretiens avec les producteurs a montré que ces produits ont détruit souvent les cultures et intoxiquent leur alimentation, raison pour laquelle, ils seraient stockés hors des maisons. La pratique d'utilisation des produits chimiques observée dans toutes les auréoles, a montré l'importance des

\section{DISCUSSIONS}

Terres cultivées dans zones riveraines: Les populations riveraines de la réserve de la biosphère Pendjari mènent pour leur subsistance l'agriculture comme principale activité. Mais les pratiques liées à cette agriculture induisent des effets néfastes sur les terres dans cette zone. II ressort des analyses que certaines pratiques positives deumeurent dans les habitudes sont : le labour à plat, le labour en billons et le sarclage manuel des champs. Le travail du sol au tracteur, dominant dans la première auréole autour de la réserve, est à la base des dégradations physiques de l'état structural par tassement, surtout lorsque le sol est humide. Cette pratique affecte la biodiversité de la réserve de biosphère Pendjari. Guy et al (2001) ont montre que les sols non labourées présenteraient, après quelques années, un état structural se caractérisant par une porosité structurale menaces qu'ils ont constituées non seulement pour une conservation durable de la réserve ; mais aussi pour les hommes. II existe cependant des recettes Endogènes biologique de traitements phytosanitaires qui ne sont pas à porter de tous et qui devraient être expérimenté et vulgariser.

plus faible qu'en situation régulièrement labourée et entraîne des risques accrus de ruissellement. Ainsi pour faire profiter aux terres riveraines de la réserve et diminuer les pressions ne serait-il pas nécessaire d'utiliser les méthodes conventionnelles de labour, moins destructrice des sols, respectant les périodes et techniques de leur utilisation.

Importance de la jachère : Dans les périphéries de la réserve, les jachères de longue durée sont presque inexistantes ce qui influence la reconstitution des sols qui n'ont plus assez de temps pour ne se régénère naturellement. C'est à cela qu'abouti l'IFS (2002) en mettant en exergue que la plupart des systèmes de cultures utilisés aboutissent à brève échéance à la dégradation des sols. Ceci se traduit par une baisse des réserves en matière organique par érosion et sur- 
minéralisation. Bahuchet, (1996) et Brady (1996), sont allés plus loin en proposant des solutions et ont estimé qu'une durée de jachère de 10 à 20 ans ou même plus sont nécessaires pour prévenir l'érosion des sols et la perte de fertilité et d'équilibre hydrique. Selon Guiran (1987), les restitutions organo-minérales au sol dans les systèmes de jachère de courte durée sont peu élevées et les systèmes agro forestiers utilisés tels la jachère améliorée et la culture en couloir restent les principales pratiques bénéfiques à l'écosystème. Au bénin en 1989, la jachère améliorée a été introduite à zouzouvou dans le couffo. Les résultats ont montré qu'en 1992, soit après 3 ans de jachère améliorée, le rendement qui était de $170 \mathrm{~kg} / \mathrm{ha}$ en 1989 , était passé a $1250 \mathrm{~kg} / \mathrm{ha}$. A la fin de la période de jachère la production ligneuse est trois fois plus élevée que la production cumulée du maïs durant la même période (Versteeg et al. ,1998).

Les engrais chimiques-minéraux (dégradation chimique des sols) : En ce qui concerne la fertilisation moins de $25 \%$ des enquêtés apporte la fertilisation organique à leur terre dans toutes les auréoles alors que $75 \%$ ont opté pour l'usage des engrais minéraux (chimiques) à des doses très variables. Cette pratique (surtout dans les deux premières auréoles) autour du Parc, épuise, appauvrie et dégrade les sols à la longue. Cela conduit à une dégradation chimique des sols de la réserve (acidification des sols). Selon les producteurs, les terres ne donnent plus les rendements sans les apports d'engrais, et les doses augmentent à chaque campagne entrainant un raccourcissement des périodes de jachère, la fertilisation minérale qui sont à la base de l'épuisement, l'acidification des sols et par conséquent la baisse des rendements (Floret et al, 1993). En effet, les producteurs continuent toujours d'apporter uniquement des engrais chimiques à leurs sols. Les quantités de fumier produites ne dépassent pas en moyenne $100 \mathrm{~kg}$ alors que le taux moyen de minéralisation d'un sol tropical est de l'ordre de

\section{CONCLUSION ET APPLICATION DES RÉSULTATS :}

La réserve de la biosphère de la Pendjari est une aire classée dont la flore et la faune constituent les principaux objets de conservation. Mais force est de constater que la conservation de ces ressources, est entravée par diverses difficultés liées à la gestion des terroirs riverains du Parc. D'où le présente étude sur limpact du mode de gestion des terroirs riverains de cette réserve pour mieux les mettre en exergue afin de les intégrer dans de futurs plans d'aménagement. Ainsi on y retient que les principaux menacent qui minent ce parc sont essentiellement la dégradation physique due à la compactation des sols essentiellement causé par
$2 \%$ par an (Saïdou, 2005). Cette minéralisation doit être compensée par un apport de matière organique la plus évoluée possible, environ 2 tonnes de fumier à $30 \%$ de matière organique par hectare par an (Saïdou, 2009). Ainsi donc, pour une efficacité, une dose de 30t/ha est recommandée (Saïdou, 2005). Aussi l'épuisement des sols est une menace à la conservation de la flore sauvage. En effet, la conservation de la faune sauvage, à l'état naturel, est sous le contrôle d'un grand nombre de facteurs qui sont à la fois liés à l'espèce et à son milieu de vie. Van der Pol (1994), Boko et Kpagbin (1997) cité par Djenontin et al (2002), abondent dans le même sens dans leur analyse en affirmant que de toute évidence, les terres agricoles connaissent dans le processus de mise en valeur et d'exploitation, une perte d'éléments nutritifs dont l'importance s'accroît au fur et à mesure que le processus dure, avec pour conséquence une chute des rendements. En général, les compensations faites sont inférieures à la perte d'éléments nutritifs engendrée par ce processus. Parmi les facteurs extrinsèques, la disponibilité en quantité et en qualité des ressources alimentaires est un facteur majeur de régulation de la dynamique des populations (McNaughton, 1979 ; Fowler, 1981). Cette disponibilité de pâturage permet d'observer un accroissement rapide et une répartition importante de la faune sauvage permettant aux aires protégées qui visent le tourisme; une vue agréable et facile des animaux. Le manque de pâturage pour la faune sauvage est très dangereux pour sa survie ainsi que pour sa conservation. Ces études viennent confirmer le problème que constitue l'utilisation incontrôlée des engrais chimiques. II urge donc de souligner l'importance d'une gestion durable de la fertilité des sols pour une conservation durable de la faune et de la flore de la réserve de la biosphère Pendjari et cela par l'utilisation modéré d'engrais chimiques.

l'utilisation des engins lourds pour la culture du coton et parfois maïs; ensuite la dégradation chimique dû à l'usage de grande quantités d'engrais chimiques surtout pour la culture du coton largement adopté. La prise en compte de ces menaces par l'organe de gestion du parc et l'adoption d'une agriculture plus biologique pourrait améliorer la conservation de cet écosystème forestier. Cette étude est un outil essentiel de la prévention des atteintes à l'environnement car à l'avenir permettra d'évaluer au préalable les effets d'une activité sur l'environnement et d'en éviter certaines conséquences dommageables. Dans un but d'approfondissement; une 
étude sur la qualité des eaux (rivière pendjari et mares) du parc devrait être envisagé pour évaluer l'état de

\section{REMERCIEMENTS}

Nous remercions très sincèrement la Direction Générale du Parc National de la Pendjari et le Centre d'Études, de Recherches et de Formation Forestières qui ont permis et

\section{REFERENCE BIBLIOGRAPHIQUE}

Aho N, Kossou D, 1997. Précis de l'agriculture tropicale. Bases et Éléments d'Applications. Les Éditions Flamboyants. 464p.

Amahowe I, Houessou L, Nago G, Nobime G, Ahokpe E, Djagoun S, Toko I, Bonou W, Gouwakinnou G, Zannou O, Agossa N, Kindjinou A, N'sera P, Chabi Oure, Nf (2013) : Dénombrement pédestre de la faune dans la Réserve de Biosphère de la Pendjari et la Réserve de Biosphère Transfrontalière du W-Bénin. CENAGREF-PAPE, Cotonou, Bénin.129p

Bahuchet S, 1996. La mer et la forêt : Ethnoécologie des populations forestières et des pêcheurs du Sud Cameroun. In : Froment A., DE Garine I., Binam Bikoi C. \& Loung.

Baldus R D, Hahn R, Kaggi D, Kaihula S, Murphree M., Mahundi C C, Roettcher K, Siege L et Zacharia M (2001). Experiences with Community Based conservation. Tanzania, Dar Es Salaam In Tanzania Wildlife Discussion Paper No. 29, p.17.

Brady N C, 1996. Alternatives to slash-and-burn: a global imperative. Agriculture, Ecosystems \& Environment $58: 3-11$

CENAGREF, 2009 : Plan d'Aménagement Participatif et de Gestion (2004-2013), Edition 5, Cotonou, Bénin.

CENAGREF, 2000: Système de Surveillance de Biosphère Pendjari.

Deldicque M, 2007: Dialogue in biosphere reserves: references, practices and experiences. In Biosphere Reserve Tecnical Notes- 2-2007, 80 $p$.

Delvingt W, Heymans J.C., Sinsin B., 1989. Guide du Parc National de la Pendjari. CECA-CEE-CEEA, $119 \mathrm{p}$.

Djenontin A J, Wennink B, Dagbenongbakin G, Ouinkoun $G$, 2002. Pratiques de gestion de fertilité dans les exploitations agricoles du Nord-Bénin, 9p. Actes du colloque, 27-31 mai 2002, Garoua, Cameroun. l'écosystème suite aux activités anthropique, gage de la conservation de la faune sauvage.

faciliter la collecte les données au niveau des villages riverains au parc Pendjari et les analyses appropriées.

Floret C, Pontanier R, 1993. Jachère en Afrique tropicale. Éditeur de la série: Malcolm Hadley. 41p.

Guy R, 2001. Fonctionnement physique des sols cultivés : labour, non-labour, structure et érosion.

Hausser Y, 2009. Mission d'évaluation des campements et zones de chasse du Bénin. CENAGREF, Cotonou, 151p.

IFS, 2002. Plan D'action Opérationnel Pour La Gestion Durable De La Fertilité Des Sols au Benin. 45p

INRA, Agronomie Laon. Les colloques de l'INRA. INRA éditions, Paris. $2 p$.

Houinato M, \& Sinsin B, 2000. La pression agro-pastorale sur la zone riveraine de la réserve de la biosphère de la Pendjari. TROPICULTURA, 2000,18,3,112-117.

McNaughton S J, Ruess RW, and Seagle S W, 1979. Large mammals and process dynamic in African ecosystem: Herbivores mammals affect primary productivity and regulate recycling balances

Roose $E, 1994$. Introduction à la gestion conservatoire de l'eau de la biomasse et la fertilité des sols. Série Bulletin pédologique $\mathrm{FAO} \mathrm{N}^{\circ} 70$.

Saidou A, 2009. Note de cours de préparation et utilisation des engrais organiques. Semestre 5 FSA/UAC/Bénin ; pp 7-9.

Saidou A, 2005. Capitalisation des expériences et pratiques de restauration de la fertilité des sols et LAE au Bénin. Rapport d'étude, PAMRAD/CTB, Natitingou Bénin. 69p.

Sebillotte M, 1982. Pratiques des agriculteurs et évolution de la fertilité du milieu. Éléments pour un jugement des systèmes de culture. B.T.I. 370/372, L1-Agro-19, pp425- 435.

Sogbohossou EA, 2008. Research on lions in Benin: review and perspectives. In Croes, B., Buij, R., longh, H.H., de, Bauer, H. (Eds.) Management and Conservation of Large Carnivores in West and Central Africa (pp. 73-80). Leiden/Maroua: CML/CEDC

Sinsin B, Tehou A, Assogbadjo A, Sogbohossou E, Mama A, Gbangboche A, Yorou S et Toko I, 2001: Dénombrement de la faune sauvage 
dans la Réserve de Biosphère de la Pendjari (Rapport technique), CENAGREF, Cotonou, Bénin, $40 \mathrm{p}$.

Tiomoko D, 2007 : Impacts des recettes de la chasse safari sur la conservation participative de la Réserve de Biosphère de la Pendjari. DEA, Université d'Abomey Calavi, $49 \mathrm{p}$.

Tiomoko D, 2014. Gestion de la Réserve de Biosphère de la Pendjari : modes de gestion et proposition d'un modèle conceptuel de durabilité, Université d'Abomey-Calavi, 2014, p. 31-32 [lire en ligne] (thèse).

Triplet $P$, Vermeulen C, 2009. Améliorer la participation des populations locales à la gestion des Aires Protégées. In Manuel de gestion des Aires Protégées d'Afrique francophone. Agence de
Coopération Culturelle et Technique \& JeanPierre de Monza, pp 228-231.

UNESCO,1995. Seconde réunion mondiale de Réserves de biosphère à Séville (Espagne). unesdoc.unesco.org/images/0016/001615/1615 36f.pdf

USAID, 2004. Fiche d'informations du bureau Afrique sur l'engrais. Rob Clausen, Regional Environmental Advisor USAID West Africa Regional Program (WARP) Accra, Ghana.2p.

Versteeg M N, Amadji F, Eteka A, Gogan A, Koudokpon V, 1998. Farmers' adoption of Mucuna spp Fallowing and Agroforetry Technologies in the Coastal Savanna of Benin. Agricultural systems, 56 : 269-287. 